\title{
Impact of Postprocedural TIMI Flow on Long-Term Clinical Outcomes in Patients with Acute Myocardial Infarction Five Year Follow-Up Results in the Corea-AMI Registry
}

\author{
Dae-Won Kim, ${ }^{1}$ MD, Sung-Ho Her, ${ }^{1}$ MD, Mahn-Won Park, ${ }^{1}$ MD, Jung Sun Cho, ${ }^{1}$ MD, \\ Tae-Seok Kim, ${ }^{1}$ MD, Hyeonjeong Kang, ${ }^{1}$ MD, Doo Sun Sim, ${ }^{2}$ MD, Young Joon Hong, ${ }^{2}$ MD, \\ Ju Han Kim, ${ }^{2}$ MD, Youngkeun Ahn, ${ }^{2}$ MD, Kiyuk Chang, ${ }^{3}$ MD, Wook-Sung Chung, ${ }^{3}$ MD, \\ Ki-Bae Seung, ${ }^{3} \mathrm{MD}$, Myung-Ho Jeong, ${ }^{2} \mathrm{MD}$ and Tai-Ho Rho, ${ }^{4} \mathrm{MD}$
}

\begin{abstract}
Summary
This study aimed to evaluate the clinical prognostic implications of postprocedural Thrombolysis in Myocardial Infarction (TIMI) flow in acute myocardial infarction patients. A total of 2796 ST-elevation myocardial infarction (STEMI) and 1720 non ST-elevation myocardial infarction (NSTEMI) patients treated in 8 hospitals affiliated with the Catholic University of Korea and Chonnam National University Hospital were analyzed. The study populations were divided according to the final TIMI flow. The primary outcome were the major adverse cardiac events (MACE), defined as a composite of cardiac deaths (CD), nonfatal myocardial infarctions (MI), and target lesion revascularization (TLR). Over a median follow-up of 3.3 years (minimum 2 to maximum 5 years), MACE and CD occurred more frequently in STEMI patients with TIMI $\leq 2$ group than those with TIMI 3 (MACE: adjusted hazard ratio [aHR], 1.962; 95\% confidence interval [CI] 1.513 to $2.546, P<0.001$, CD: aHR, 3.154, CI 2.308 to $4.309, P<0.001$ ). However, there was no significant difference between the two subgroups in NSTEMI (aHR, 0.932 ; 95\% CI 0.586 to $1.484, P=0.087$ ). In STEMI patients, good postprocedural TIMI flow after PCI was associated with favorable clinical outcomes. And the effect of poor TIMI flow in STEMI was on death, not the components of MACE. Meanwhile, postprocedural TIMI flow had no effect on long-term outcomes in NSTEMI patients.
\end{abstract}

Key words: STEMI, NSTEMI, MACE

(Int Heart J 2017; 58: 674-685)

$\mathrm{R}$ apid reestablishment of normal (Thrombolysis in Myocardial Infarction [TIMI] grade 3) flow in the infarct-related artery has contributed to the advantages of reperfusion therapies for ST elevation myocardial infarction (STEMI). ${ }^{1,2}$ Currently, percutaneous coronary intervention (PCI) is the first line treatment for patients with STEMI ${ }^{3,4)}$ Unfortunately, reopening of a culprit coronary artery after adequate reperfusion therapy does not always improve tissue perfusion. It is called suboptimal coronary flow defined as TIMI flow grade $\leq 2$. It is well known that suboptimal coronary flow has been associated with poor prognosis in STEMI patients. ${ }^{4.8}$ In detail, it is related to one-month left ventricular dysfunction, increased 1-month or 1-year mortality risk according to post-PCI TIMI flow grade, composite of death, repeat myocardial infarction (MI), target vessel revascularization at 30 days, and increased in-hospital long-term cardiovas- cular mortalities. Meanwhile, it remains unclear whether postprocedural coronary flow after PCI is related with long-term clinical outcomes in non ST-segment elevation myocardial infarction (NSTEMI). Thus, the aim of this multicenter, retrospective, observational study is to evaluate the long-term prognostic role of postprocedural TIMI flow after PCI using a large cohort of acute myocardial infarction.

\section{Editorial p.645}

\section{Methods}

Participants: A total of 5025 patients with either STEMI or NSTEMI who had admitted at 9 major cardiovascular centers came under the Convergent Registry of Catholic

From the ${ }^{1}$ Division of Cardiology, Daejeon St. Mary's Hospital, College of Medicine, The Catholic University of Korea, DaeJeon, ${ }^{2}$ Cardiovascular Center, Chonnam National University Hospital, Chonnam National University, Gwangju, ${ }^{3}$ Division of Cardiology, Seoul St. Mary's Hospital, College of Medicine, The Catholic University of Korea and ${ }^{4}$ Division of Cardiology, Seoul St. Paul's Hospital, College of Medicine, The Catholic University of Korea, Seoul, Republic of Korea.

This study was supported by Yuhan Corporation, but the assistance of the company had no role in the design and conduct of the study; the collection, analysis, and interpretation of the data; or the preparation, review, or approval of the manuscript.

Address for correspondence: Sung-Ho Her, MD, Division of Cardiology, Daejeon St. Mary's Hospital, College of Medicine, The Catholic University of Korea, 64 Daeheung-ro, Jung-gu, Daejeon 34943, Republic of Korea. E-mail: hhhsungho@ naver.com

Received for publication September 12, 2016. Revised and accepted November 13, 2016.

Released in advance online on J-STAGE September 30, 2017.

doi: 10.1536/ihj.16-448

All rights reserved by the International Heart Journal Association. 


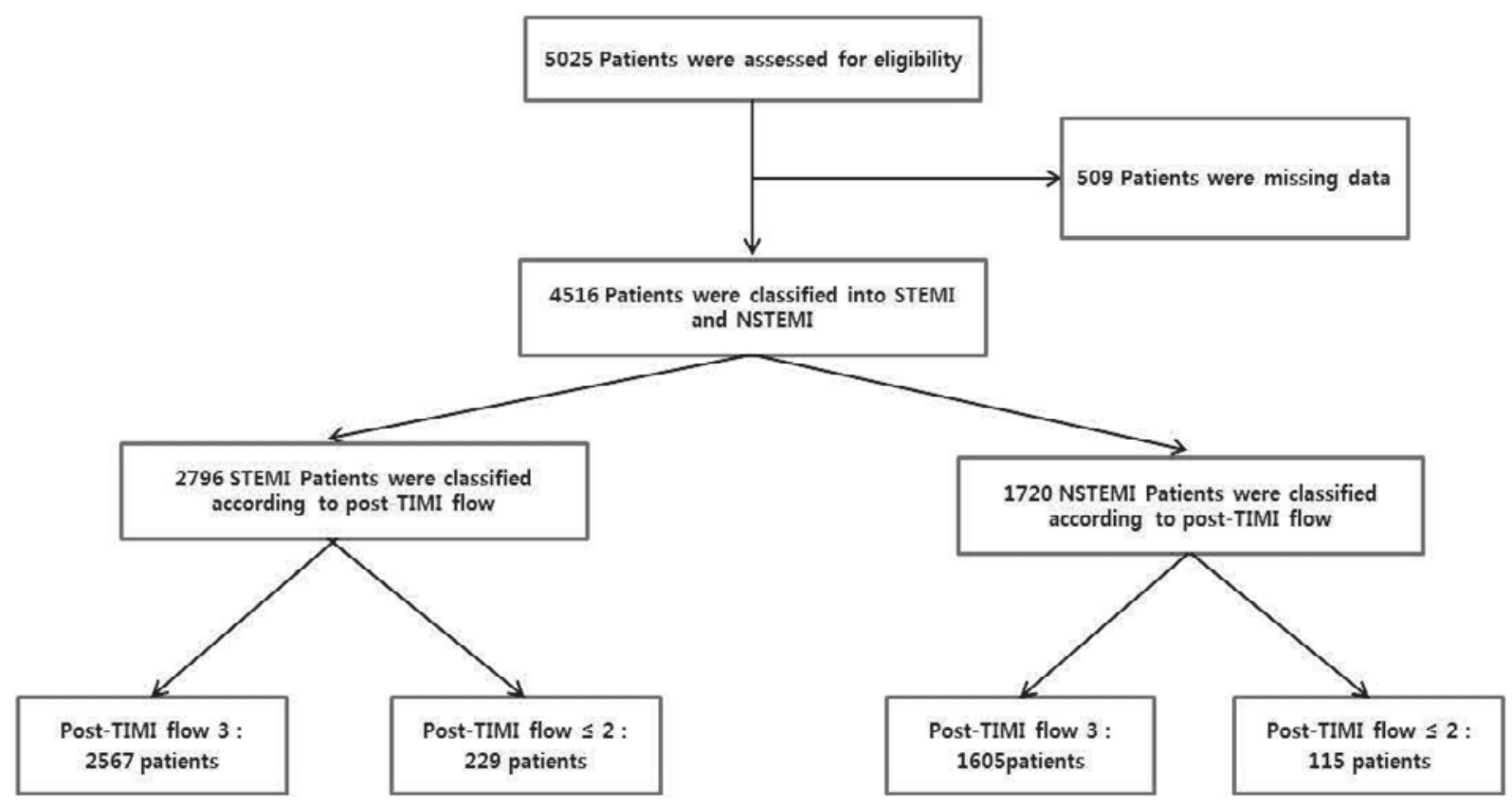

Figure 1. Study population

and Chonnam University for Acute Myocardial Infarction (COREA-AMI) from January 2004 to July 2012. A median follow-up duration was 3.3 years (minimum 2 to maximum 5 years) and the follow-up rate in 5 years was $21.2 \%$.

This large observational registry was designed to evaluate clinical outcomes of patients with acute MI including both STEMI and NSTEMI, and included demographic, clinical, and angiographic data with short-term and long-term clinical outcome data. Of 5025 patients, 509 patients without post-TIMI flow data were excluded and 4516 patients were included in the analyses.

Among 4516 patients, 2796 and 1720 patients were diagnosed with STEMI and NSTEMI, respectively, and underwent successful PCI. STEMI was diagnosed by the presence of chest pain lasting for more than $20 \mathrm{~min}$ in association with electrocardiographic changes (ST-segment elevation of $\geq 1 \mathrm{~mm}$ in at least two extremity electrocardiographic leads or $\geq 2 \mathrm{~mm}$ in at least contiguous precordial leads, or new-onset left-bundle branch block). NSTEMI was defined as increased cardiac markers with symptoms compatible with myocardial ischemia in the absence of ST-elevation on the index ECG. ${ }^{9)}$ Meanwhile, in baseline characteristics, smoking included current smokers and ex-smokers and renal failure was defined with diagnosed chronic renal failure, creatinine $>2.0 \mathrm{mg} / \mathrm{dL}$ and patients on dialysis.

Percutaneous coronary intervention procedure and medical treatment: Coronary angiography and PCI were performed according to current standard guidelines. A definition of successful PCI required the following: 1) Angiographic success: consensus definition prior to the use of stents was the achievement of a minimum stenosis diameter reduction to $<50 \%$ in the presence of grade 3 TIMI flow (assessed by angiography). However, with the advent of advanced adjunct technology, including coronary stents, a minimum stenosis diameter reduction to < $20 \%$ has been the clinical benchmark of an optimal angiographic result. 2) Procedural success: A successful PCI should achieve angiographic success without in-hospital major clinical complications (e.g., death, MI, emergency coronary artery bypass surgery) during hospitalization. 3) Clinical success: In the short term, a clinically successful PCI includes anatomic and procedural success with relief of signs and/or symptoms of myocardial ischemia after the patient recovers from the procedure. The long-term clinical success requires that the short-term clinical success remains durable and that the patient has persistent relief of signs and symptoms of myocardial ischemia for more than 6 months after the procedure. ${ }^{10)}$ Antiplatelet therapy and administration of periprocedural anticoagulation were carried out in accordance with standard regimens. All patients were prescribed aspirin (loading dose, $200 \mathrm{mg}$ ) plus clopidogrel (loading dose, 300 or $600 \mathrm{mg}$ ) before or during PCI. After the procedure, aspirin (100$200 \mathrm{mg} /$ day) was maintained indefinitely. Patients with drug-eluting stents were prescribed clopidogrel $(75 \mathrm{mg}$ / day) for at least 12 months while patients with bare-metal stents were prescribed clopidogrel for at least 1 month. Other cardiac medications were administered at the discretion of treating physicians.

The completeness of restoration of the epicardial blood flow after recanalization of infarct-related artery has been quantified with the Thrombolysis in Myocardial Infarction (TIMI) grading system, which was categorized by each operator. ${ }^{11)}$ All patients were classified into 2 groups according to the postprocedural TIMI flow which reflects anterograde blood flow of the infarct vessel (Figure 1).

Study End-Points: The primary end-point was a composite of cardiac death (CD), nonfatal myocardial infarction 
(MI), and target lesion revascularization (TLR). CD was defined as any death due to a proximate cardiac cause such as MI, low-output failure, arrhythmia, and unwitnessed death. MI was defined as newly developed Q wave, raised CK-MB, Tn-I or T above the normal ranges, typical ischemic symptom with accompanied ST-segment changes in 2 or more contiguous leads on the 12-lead ECG.

Immediate postprocedural and in-hospital events were recorded. After the discharge, the patients were followed up in the out-patient clinics or by telephone 1,6 , and 12 months after the procedure and then annually. Information on censored survival data and death was obtained from hospital records or through telephone calls to relatives of the patients. All clinical outcomes of interest were confirmed by source documents and were centrally adjudicated by a local events committee at the Cardiovascular Center of Seoul St. Mary's Hospital by an independent group of clinicians unaware of patient status.

Information about death was validated by records from the National Population Registry of the Korea National Statistical Office using a unique personal identification number for each patient. The study protocol was approved by the institutional review board of each participating institution, and was conducted according to the Declaration of Helsinki. Each patient was provided with written informed consent.

Statistical analyses: Student $t$-test and $\chi^{2}$ test (or the Fisher exact test) were used to compare the means and proportion of baseline clinical and angiographic characteristics between 2 groups. Continuous variables were expressed as the mean \pm standard deviation and categorical variables were expressed as a number (percentage).

Cox proportional hazard model was used to estimate the hazard ratio (HR) and $95 \%$ confidence interval (CI) for the statistical impact of good postprocedural TIMI flow after PCI on the clinical events.

The incidence of each adverse event was estimated at 12, 24, 48, and 60 months, displayed with Kaplan-Meier curves and compared with the log-rank test.

Propensity score (PS) matching was used to identify a cohort of patients with similar baseline characteristics and to address bias and confounds in this observational study. Propensity score was computed by nonparsimonious multiple logistic regression analysis (cstatistics $=0.637$ in STEMI groups, 0.707 in NSTEMI groups). All baseline characteristics described in Table I were matched by the variables that showed differences in the overall population. Matching was performed with the use of a 1:1 nearest neighbor matching, from initial 7 to 1 digit. We confirmed the model reliability with goodnessof-fit test (0.437 in STEMI group, 0.301 in NSTEMI group).

In the matched cohort, paired comparisons were performed with the use of McNemar's test for binary variables and a paired Student's $t$-test for continuous variables. In the PS matching population, an elevation in the risk of outcomes was compared using a conditional Cox regression model.

Meanwhile, multivariable logistic regression analyses were carried out to identify independent predictors associ- ated with poor postinterventional TIMI flow after PCI. All of the variables in Table I were included and analyzed to perform univariable logistic regression analysis. On the basis of the variables that were significant $(P<0.05)$ according to univariable logistic regression analysis, a multivariable logistic regression model was constructed.

A two-tailed $P$-value of $<0.05$ was considered statistically significant. All statistical analyses were performed using a Statistical Analysis Software (SAS, version 9.2, SAS Institute, Cary, NC, USA).

\section{Results}

Baseline Characteristics of the overall study population: Baseline and angiographic characteristics are presented according to the existence of ST elevation (Table I). A total of 4516 patients included 2785 patients with STEMI and 1720 patients with NSTEMI. Their mean age was 62.6 years. In the patient group of TIMI flow grade 3 in the overall population, $71.5 \%$ were men, $31.2 \%$ had diabetes, and $49.8 \%$ had history of hypertension. In the patient group of TIMI flow grade $\leq 2,70.3 \%$ were men, $34 \%$ had diabetes, and $52.6 \%$ had hypertension. Their mean age was 63.6 years. There were no significant statistical differences between the two groups. The patient group of TIMI flow $\leq 2$ were lighter smokers and included more Killip class IV and more extensive coronary artery disease (CAD). Their left ventricular ejection fraction (LVEF) on admission was lower, and total cholesterol and high density lipoprotein (HDL) cholesterol were also lower, whereas low density lipoprotein (LDL) cholesterol and baseline high-sensitivity C-reactive protein (hsCRP) were higher. In addition, in the patient group of TIMI flow $\leq 2$, the left anterior descending artery (LAD) was the most frequent culprit artery $(51.7 \%)$, followed by right coronary artery (RCA, 31.4\%) and left circumflex artery (LCX, 11\%), and the same incidence pattern was shown in the patient group of TIMI flow 3 (LAD, RCA, LCX $47.9 \%, 33.8 \%, 16.2 \%)$. These results were consistent in the comparison analysis of the patients with STEMI and NSTEMI. In both STEMI and NSTEMI groups, the incidence of Killip IV was higher and the patients tended to be lighter smokers and had lower total cholesterol when TIMI flow was $\leq 2$. The incidence pattern of culprit vessel was also comparable to that of the overall population. However, NSTEMI patients with TIMI flow $\leq 2$ had more extensive CAD, lower fasting blood glucose, and lower LDL cholesterol, whereas STEMI patients with TIMI flow $\leq 2$ had lower LVEF, lower HDL cholesterol, and higher hsCRP.

Baseline Characteristics of the PS-Matched population: After PS matching, 344 patients (overall population), 115 patients (STEMI), and 299 patients (NSTEMI) were allocated into each group (Table II). In the matched cohort, there were no significant differences in baseline characteristics.

Clinical outcomes of the overall population: The median follow-up duration was 5 years. Among the patients with STEMI, the cumulative rates of primary end-point including CD, MI, and TLR were significantly higher in the suboptimal postcoronary flow group (TIMI $\leq 2$ ) than 
Table I. Baseline Characteristics according to Postintervention TIMI Flow Grade

\begin{tabular}{|c|c|c|c|c|c|c|c|c|c|}
\hline \multirow[b]{2}{*}{ Characteristic } & \multicolumn{3}{|c|}{ Overall } & \multicolumn{3}{|c|}{ NSTEMI } & \multicolumn{3}{|c|}{ STEMI } \\
\hline & $\begin{array}{c}\text { TIMI } \\
\text { flow } 3 \\
(n=4172)\end{array}$ & $\begin{array}{c}\text { TIMI } \\
\text { flow } \leq 2 \\
(n=344)\end{array}$ & $P$ & $\begin{array}{c}\text { TIMI } \\
\text { flow } 3 \\
(n=1605)\end{array}$ & $\begin{array}{c}\text { TIMI } \\
\text { flow } \leq 2 \\
(n=115)\end{array}$ & $P$ & $\begin{array}{c}\text { TIMI } \\
\text { flow } 3 \\
(n=2567)\end{array}$ & $\begin{array}{c}\text { TIMI } \\
\text { flow } \leq 2 \\
(n=229)\end{array}$ & $P$ \\
\hline Age (years) & $62.6 \pm 12.5$ & $63.6 \pm 12.6$ & 0.164 & $63.9 \pm 12.3$ & $64.6 \pm 11.1$ & 0.552 & $61.8 \pm 12.6$ & $63.1 \pm 13.3$ & 0.143 \\
\hline Gender (male) $(\%)$ & $2985(71.5)$ & $242(70.3)$ & 0.636 & $1096(68.3)$ & $77(67.0)$ & 0.767 & $1889(73.6)$ & $165(72.1)$ & 0.614 \\
\hline \multicolumn{10}{|l|}{ Killip classification $(\%)$} \\
\hline I & $3283(78.7)$ & $246(71.5)$ & $<0.001$ & $1283(79.9)$ & $84(73.0)$ & 0.048 & $2000(77.9)$ & $162(70.7)$ & $<0.001$ \\
\hline II & $423(10.1)$ & $45(13.1)$ & & $161(10.0)$ & $19(16.5)$ & & $262(10.2)$ & $26(11.4)$ & \\
\hline III & $242(5.8)$ & $14(4.1)$ & & $106(6.6)$ & $5(4.3)$ & & $136(5.3)$ & $9(3.9)$ & \\
\hline IV & $224(5.4)$ & $39(11.3)$ & & $55(3.4)$ & $7(6.1)$ & & $169(6.6)$ & $32(14.0)$ & \\
\hline Hypertension (\%) & $2078(49.8)$ & $181(52.6)$ & 0.317 & $868(54.1)$ & $67(58.3)$ & 0.385 & $1210(47.1)$ & $114(49.8)$ & 0.442 \\
\hline Diabetes $(\%)$ & $1300(31.2)$ & $117(34.0)$ & 0.273 & $543(33.8)$ & $49(42.6)$ & 0.056 & $757(29.5)$ & $68(29.7)$ & 0.948 \\
\hline Smoking $(\%)$ & $2443(58.6)$ & $161(46.8)$ & $<0.001$ & $889(55.4)$ & $47(40.9)$ & 0.003 & $1554(60.5)$ & $114(49.8)$ & 0.002 \\
\hline Family history of CAD (\%) & $230(5.5)$ & $16(4.7)$ & 0.499 & $82(5.1)$ & $8(7.0)$ & 0.390 & $148(5.8)$ & $8(3.5)$ & 0.151 \\
\hline Renal failure (\%) & $205(4.9)$ & $19(5.5)$ & 0.617 & $110(6.9)$ & $11(9.6)$ & 0.272 & $95(3.7)$ & $8(3.5)$ & 0.873 \\
\hline Extent of CAD (multi vessel) $(\%)$ & $2200(52.7)$ & $207(60.2)$ & 0.008 & $928(57.8)$ & $87(75.7)$ & $<0.001$ & $1272(49.6)$ & $120(52.4)$ & 0.409 \\
\hline \multicolumn{10}{|l|}{ Culprit vessel (\%) } \\
\hline Left anterior descending artery & $2000(47.9)$ & $178(51.7)$ & $<0.001$ & $707(44.0)$ & $52(45.2)$ & $<0.001$ & $1293(50.4)$ & $126(55.0)$ & 0.010 \\
\hline Left circumflex artery & $676(16.2)$ & $38(11.0)$ & & $386(24.0)$ & $16(13.9)$ & & $290(11.3)$ & $22(9.6)$ & \\
\hline Right coronary artery & $1412(33.8)$ & $108(31.4)$ & & $471(29.3)$ & $37(32.2)$ & & $941(36.7)$ & $71(31.0)$ & \\
\hline Left main & $84(2.0)$ & $20(5.8)$ & & $41(2.6)$ & $10(8.7)$ & & $43(1.7)$ & $10(4.4)$ & \\
\hline \multicolumn{10}{|l|}{ Post TIMI flow of culprit vessel (\%) } \\
\hline 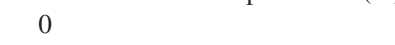 & & $195(56.7)$ & & & $92(80.0)$ & & & $103(45.0)$ & \\
\hline I & & $28(8.1)$ & & & $5(4.3)$ & & & $23(10.0)$ & \\
\hline II & & $121(35.2)$ & & & $18(15.7)$ & & & $103(45.0)$ & \\
\hline III & $4172(100.0)$ & $0(0.0)$ & & $1605(100.0)$ & $0(0.0)$ & & $2567(100.0)$ & $0(0.0)$ & \\
\hline Ejection fraction $(\%)$ & $53.0 \pm 10.7$ & $50.7 \pm 11.4$ & $<0.001$ & $53.9 \pm 11.8$ & $53.0 \pm 10.1$ & 0.077 & $53.3 \pm 11.6$ & $49.4 \pm 11.8$ & $<0.001$ \\
\hline FBS (mg/dL) & $146.4 \pm 67.1$ & $164.3 \pm 83.7$ & 0.536 & $164.5 \pm 78.5$ & $145.7 \pm 66.9$ & 0.012 & $165.4 \pm 78.5$ & $173.8 \pm 89.8$ & 0.503 \\
\hline Creatinine $(\mathrm{mg} / \mathrm{dL})$ & $1.3 \pm 1.0$ & $1.2 \pm 0.8$ & 0.265 & $1.1 \pm 1.0$ & $1.3 \pm 1.0$ & 0.419 & $1.1 \pm 0.9$ & $1.2 \pm 0.6$ & 0.344 \\
\hline Total cholesterol (mg/dL) & $172.4 \pm 41.2$ & $172.1 \pm 40.8$ & $<0.001$ & $181.3 \pm 41.8$ & $171.2 \pm 40.4$ & 0.013 & $180.4 \pm 41.0$ & $172.6 \pm 41.1$ & 0.007 \\
\hline Triglyceride (mg/dL) & $122.2 \pm 80.3$ & $111.8 \pm 72.2$ & 0.056 & $122.2 \pm 89.4$ & $121.9 \pm 79.0$ & 0.547 & $119.2 \pm 86.3$ & $106.3 \pm 67.9$ & 0.065 \\
\hline HDL cholesterol (mg/dL) & $42.2 \pm 12.4$ & $41.5 \pm 10.9$ & 0.010 & $43.0 \pm 11.6$ & $42.2 \pm 12.6$ & 0.564 & $43.1 \pm 11.6$ & $41.1 \pm 9.8$ & 0.002 \\
\hline LDL cholesterol (mg/dL) & $105.8 \pm 36.3$ & $107.7 \pm 36.0$ & 0.011 & $113.8 \pm 37.8$ & $103.4 \pm 36.5$ & 0.022 & $113.4 \pm 36.8$ & $110.1 \pm 35.6$ & 0.157 \\
\hline hsCRP (mg/L) & $2.9 \pm 4.9$ & $2.9 \pm 5.0$ & 0.027 & $2.3 \pm 4.0$ & $2.9 \pm 5.0$ & 0.194 & $2.3 \pm 4.0$ & $2.9 \pm 5.0$ & 0.074 \\
\hline HbAlc $(\%)$ & $6.6 \pm 1.6$ & $6.6 \pm 1.6$ & 0.908 & $6.6 \pm 1.5$ & $6.6 \pm 1.5$ & 0.560 & $6.6 \pm 1.5$ & $6.6 \pm 1.6$ & 0.757 \\
\hline $\mathrm{Hb}(\mathrm{g} / \mathrm{dL})$ & $13.0 \pm 2.2$ & $13.5 \pm 2.0$ & 0.066 & $13.2 \pm 2.4$ & $13.0 \pm 2.1$ & 0.807 & $13.4 \pm 2.3$ & $13.7 \pm 1.9$ & 0.058 \\
\hline Aspirin (\%) & 4157 (99.6) & $341(99.1)$ & 0.153 & $1599(99.6)$ & $114(99.1)$ & 0.385 & 2558 (99.6) & $227(99.1)$ & 0.226 \\
\hline Clopidogrel (\%) & 4147 (99.4) & $339(98.5)$ & 0.073 & $1592(99.2)$ & $114(99.1)$ & $>0.999$ & 2555 & $225(98.3)$ & 0.036 \\
\hline Statin $(\%)$ & $3581(85.8)$ & $303(88.1)$ & 0.248 & $1363(84.9)$ & $108(93.9)$ & 0.008 & $2218(86.4)$ & $195(85.2)$ & 0.598 \\
\hline
\end{tabular}

Data are presented as mean $\pm \mathrm{SD}$, median (interquartile range), and number (percentage) where appropriate. CAD indicates coronary artery disease; TIMI, Thrombolysis in Myocardial Infarction; FBS, fasting blood sugar; HDL, high-density lipoprotein; LDL, low-density lipoprotein; Hs$\mathrm{CRP}$, high sensitive-C reactive protein; HbAlc, glycated hemoglobin; and $\mathrm{Hb}$, hemoglobin.

the reflow postprocedural coronary flow group (TIMI 3) at 5 years $(407$ [15.9\%] versus 66 [28.8\%], $P<0.001$, Table III). Moreover, the incidence of CD among all individuals at 5 years was significantly higher in the STEMI patient with TIMI flow $\leq 2(P<0.001$, Table III). There was no significant difference in the primary end point of NSTEMI between the subgroups of TIMI flow $\leq 2$ and TIMI 3. At 5 years, there was no significant difference in the cumulative rates of major cardiac adverse events (MACE) between the suboptimal postcoronary flow group $(\mathrm{TIMI} \leq 2)$ and the reflow group (TIMI 3) (279 [17.4\%] versus $19[16.5 \%], P=0.814)$ as well as CD $(114[7.1 \%]$ versus 11 [9.6\%], $P=0.326$ at 5 years). Multivariate Cox regression analysis revealed that postprocedural suboptimal coronary flow in STEMI is a predictor for these events (primary end-points, HR 1.962 [1.513-2.546], $P<$
0.001 at 5 years; CD, HR 3.154 [2.308-4.309], $P<0.001$ at 5 years, respectively, Table III). However, postprocedural suboptimal coronary flow in NSTEMI was not found to be a predictor for any events (primary endpoints, HR 0.932 [0.586-1.484], $P=0.087$ at 5 years; CD, HR 1.330 [0.716-2.471], $P=0.366$ at 5 years; MI, HR 0.430 [0.059-3.148], $P=0.406$ at 5 years; TLR, HR 0.708 [0.348-1.439], $P=0.340$ at 5 years, respectively, Table III).

Clinical outcomes of the PS-Matched population: In the PS-matched population, the overall findings were consistent. The primary end-point occurred in $66(28.8 \%)$ of STEMI patients with TIMI flow $\leq 2$ and 41 (17.9\%) of STEMI patients with TIMI flow $3(P=0.005)$ during 5 years. The incidence of CD was shown more frequently in STEMI patients with TIMI flow $\leq 2(P=0.002)$. How- 
Table II. Baseline Characteristics after Propensity-Score Matching according to Postintervention TIMI Flow Grade

\begin{tabular}{|c|c|c|c|c|c|c|c|c|c|}
\hline \multirow{2}{*}{$\begin{array}{c}\text { PSM } \\
\text { Characteristic }\end{array}$} & \multicolumn{3}{|c|}{ Overall } & \multicolumn{3}{|c|}{ NSTEMI } & \multicolumn{3}{|c|}{ STEMI } \\
\hline & $\begin{array}{c}\text { TIMI } \\
\text { flow } 3 \\
(n=344)\end{array}$ & $\begin{array}{c}\text { TIMI } \\
\text { flow } \leq 2 \\
(n=344)\end{array}$ & $P$ & $\begin{array}{c}\text { TIMI } \\
\text { flow } 3 \\
(n=115)\end{array}$ & $\begin{array}{c}\text { TIMI } \\
\text { flow } \leq 2 \\
(n=115)\end{array}$ & $P$ & $\begin{array}{c}\text { TIMI } \\
\text { flow } 3 \\
(n=229)\end{array}$ & $\begin{array}{c}\text { TIMI } \\
\text { flow } \leq 2 \\
(n=229)\end{array}$ & $P$ \\
\hline Age (years) & $64.1 \pm 12$ & $63.6 \pm 12.6$ & 0.579 & $66 \pm 12.9$ & $64.6 \pm 11.1$ & 0.309 & $63.4 \pm 12.4$ & $63.1 \pm 13.3$ & 0.756 \\
\hline Gender (male) $(\%)$ & $228(66.3)$ & $242(70.3)$ & 0.272 & $80(69.6)$ & $77(67.0)$ & 0.788 & $157(68.6)$ & $165(72.1)$ & 0.484 \\
\hline \multicolumn{10}{|l|}{ Killip classification $(\%)$} \\
\hline I & $246(71.5)$ & $246(71.5)$ & 0.113 & $95(82.6)$ & $84(73.0)$ & 0.204 & $154(67.2)$ & $162(70.7)$ & 0.720 \\
\hline II & $37(10.8)$ & $45(13.1)$ & & $9(7.8)$ & $19(16.5)$ & & $32(14.0)$ & $26(11.4)$ & \\
\hline III & $17(4.9)$ & $14(4.1)$ & & $6(5.2)$ & $5(4.3)$ & & $8(3.5)$ & $9(3.9)$ & \\
\hline IV & $44(12.8)$ & $39(11.3)$ & & $5(4.3)$ & $7(6.1)$ & & $35(15.3)$ & $32(14.0)$ & \\
\hline Hypertension $(\%)$ & $181(52.6)$ & $181(52.6)$ & $>0.999$ & $65(56.5)$ & $67(58.3)$ & 0.894 & $113(49.3)$ & $114(49.8)$ & $>0.999$ \\
\hline Diabetes (\%) & $124(36.0)$ & $117(34.0)$ & 0.623 & $46(40)$ & $49(42.6)$ & 0.780 & $65(28.4)$ & 68 (29.7) & 0.836 \\
\hline Smoking $(\%)$ & $169(49.1)$ & $161(46.8)$ & 0.533 & $47(40.9)$ & $47(40.9)$ & $>0.999$ & $115(50.2)$ & $114(49.8)$ & $>0.999$ \\
\hline Family history of CAD (\%) & $14(4.1)$ & $16(4.7)$ & 0.851 & $6(5.2)$ & $8(7.0)$ & 0.791 & $13(5.7)$ & $8(3.5)$ & 0.383 \\
\hline Renal failure (\%) & $21(6.1)$ & $19(5.5)$ & 0.860 & $7(6.1)$ & $11(9.6)$ & 0.481 & $11(4.8)$ & $8(3.5)$ & 0.629 \\
\hline Extent of CAD (multi vessel) (\%) & $212(61.6)$ & 207 (60.2) & 0.747 & $92(80.0)$ & $87(75.7)$ & 0.332 & $105(45.9)$ & $120(52.4)$ & 0.203 \\
\hline \multicolumn{10}{|l|}{ Culprit vessel (\%) } \\
\hline Left anterior descending & $174(50.6)$ & $178(51.7)$ & 0.867 & $53(46.1)$ & $52(45.2)$ & 0.433 & $128(55.9)$ & $126(55.0)$ & 0.517 \\
\hline Left circumflex artery & $33(9.6)$ & $38(11.0)$ & & $19(16.5)$ & $16(13.9)$ & & $22(9.6)$ & $22(9.6)$ & \\
\hline Right coronary artery & $116(33.7)$ & $108(31.4)$ & & $33(28.7)$ & $37(32.2)$ & & $73(31.9)$ & $71(31.0)$ & \\
\hline Left main & $21(6.1)$ & $20(5.8)$ & & $10(8.7)$ & $10(8.7)$ & & $6(2.6)$ & $10(4.4)$ & \\
\hline \multicolumn{10}{|l|}{ Post TIMI flow of culprit vessel (\%) } \\
\hline 0 & $0(0.0)$ & $195(56.7)$ & & $0(0.0)$ & $92(80.0)$ & & $0(0.0)$ & $103(45.0)$ & \\
\hline I & $0(0.0)$ & $28(8.1)$ & & $0(0.0)$ & $5(4.3)$ & & $0(0.0)$ & $23(10.0)$ & \\
\hline II & $0(0.0)$ & $121(35.2)$ & & $0(0.0)$ & $18(15.7)$ & & $0(0.0)$ & $103(45.0)$ & \\
\hline III & $344(100.0)$ & $0(0.0)$ & & $115(100.0)$ & $0(0.0)$ & & $229(100.0)$ & $0(0.0)$ & \\
\hline Ejection fraction (\%) & $51.1 \pm 12.7$ & $50.8 \pm 11.6$ & 0.681 & $53.9 \pm 11.8$ & $53.0 \pm 10.1$ & 0.671 & $50.4 \pm 11.0$ & $49.4 \pm 11.8$ & 0.419 \\
\hline FBS (mg/dL) & $166.6 \pm 77.7$ & $161.8 \pm 83$ & 0.425 & $163.6 \pm 80.9$ & $146.4 \pm 67.1$ & 0.085 & $174.7 \pm 83.0$ & $169.5 \pm 89.1$ & 0.512 \\
\hline Creatinine (mg/dL) & $1.2 \pm 1.0$ & $1.2 \pm 0.8$ & 0.577 & $1.2 \pm 0.9$ & $1.3 \pm 1.0$ & 0.301 & $1.1 \pm 0.7$ & $1.2 \pm 0.6$ & 0.116 \\
\hline Total cholesterol (mg/dL) & $172.4 \pm 39.6$ & $172.6 \pm 41$ & 0.926 & $169.4 \pm 38$ & $172.4 \pm 41.2$ & 0.449 & $178.4 \pm 45.8$ & $172.8 \pm 41.0$ & 0.178 \\
\hline Triglyceride (mg/dL) & $113.6 \pm 91.6$ & $114.1 \pm 74.3$ & 0.936 & $115.2 \pm 75.1$ & $122.2 \pm 80.3$ & 0.493 & $121.7 \pm 93.2$ & $110.0 \pm 70.9$ & 0.156 \\
\hline HDL cholesterol (mg/dL) & $42.7 \pm 11.9$ & $41.4 \pm 10.8$ & 0.123 & $42.6 \pm 12$ & $42.2 \pm 12.4$ & 0.814 & $40.9 \pm 11.0$ & $40.9 \pm 9.9$ & 0.995 \\
\hline LDL cholesterol (mg/dL) & $107.7 \pm 33.3$ & $109.1 \pm 35.8$ & 0.579 & $105.4 \pm 31.8$ & $105.9 \pm 36.3$ & 0.888 & $113.3 \pm 37.6$ & $110.6 \pm 35.6$ & 0.456 \\
\hline hsCRP (mg/L) & $2.6 \pm 4.3$ & $2.9 \pm 4.9$ & 0.375 & $2.4 \pm 3.2$ & $2.9 \pm 4.9$ & 0.333 & $2.5 \pm 4.9$ & $2.9 \pm 4.9$ & 0.448 \\
\hline HbAlc $(\%)$ & $6.6 \pm 1.6$ & $6.6 \pm 1.6$ & 0.931 & $6.6 \pm 1.5$ & $6.6 \pm 1.5$ & 0.560 & $6.6 \pm 1.5$ & $6.6 \pm 1.6$ & 0.757 \\
\hline $\mathrm{Hb}(\mathrm{g} / \mathrm{dL})$ & $13.1 \pm 2.4$ & $13.5 \pm 2.0$ & 0.050 & $13.1 \pm 2.2$ & $13.0 \pm 2.1$ & 0.804 & $13.7 \pm 2.2$ & $13.7 \pm 1.9$ & 0.688 \\
\hline Aspirin (\%) & 342 (99.4) & 341 (99.1) & $>0.999$ & $114(99.1)$ & $114(99.1)$ & $>0.999$ & $229(100.0)$ & $227(99.1)$ & 0.500 \\
\hline Clopidogrel (\%) & $342(99.4)$ & 339 (98.5) & 0.453 & $115(100.0)$ & $114(99.1)$ & $>0.999$ & $229(100.0)$ & 225 & 0.125 \\
\hline Statin $(\%)$ & $294(85.5)$ & $303(88.1)$ & 0.336 & $106(92.2)$ & $108(93.9)$ & 0.727 & $195(85.2)$ & $195(85.2)$ & 0.328 \\
\hline
\end{tabular}

Data are presented as mean $\pm \mathrm{SD}$, median (interquartile range), and number (percentage) where appropriate. CAD indicates coronary artery disease; TIMI, Thrombolysis in Myocardial Infarction; FBS, fasting blood sugar; HDL, high-density lipoprotein; LDL, low-density lipoprotein; Hs$\mathrm{CRP}$, high sensitive-C reactive protein; HbAlc, glycated hemoglobin; and $\mathrm{Hb}$, hemoglobin.

ever, there were no significant differences in the primary outcomes $(P>0.999)$ and in the major cardiac events (CD, $P>0.999$; MI, $P>0.999$; TLR, $P>0.999$ ) between NSTEMI patients with TIMI flow $\leq 2$ and NSTEMI patients with TIMI flow 3. The cumulative rate of the primary end-point was significantly higher in the STEMI patients with TIMI flow $\leq 2(28.8 \%$ versus $17.9 \%, P=$ 0.005 , HR 1.692 [1.145-2.498], $P=0.008$, Table III). The occurrence of $\mathrm{CD}$ adverse event was also significantly higher in the STEMI patients with TIMI flow $\leq 2(21.8 \%$ versus $11.4 \%, P=0.002$, HR 1.975 [1.229-3.173], $P=$ 0.005 , Table III). On the other hand, there were no significant differences in the PS-matched population of the NSTEMI patients (primary end-points, HR 0.931 [0.4961.744], $P=0.822$ at 5 years; CD, HR 0.918 [0.4052.078], $P=0.837$ at 5 years; MI, HR 0.775 [0.048-
12.412], $P=0.857$ at 5 years; TLR, HR 0.955 [0.3582.548], $P=0.926$ at 5 years, respectively, Table III).

Kaplan-Meier and landmark analysis in the PSmatched population: The Kaplan-Meier curve indicated a significantly higher risk for primary end-point in the STEMI patients with TIMI flow $\leq 2$ during 5 years (event-free survival rate: $66.9 \%$ versus $80.4 \%, P<0.001$, Figure 2). The 3-month landmark analysis revealed that there was no significantly higher risk for the primary endpoint in the STEMI patients with TIMI flow $\leq 2$ after 3 months $(P=0.846$, Figure 4). Figure $2 \& 3$ showed Kaplan-Meier curves for the incidence of MACE and CD in both STEMI and NSTEMI patients during 5 years. Although the CD-free survival rate for 5 years was significantly lower in the STEMI patients with TIMI flow $\leq 2$ (74.5\% versus $90.4 \% ; P<0.001$ ), the 5-year landmark 
Table III. Five-Year Clinical Outcomes in MI Patients before Matching and Propensity Score Matched Populations

\begin{tabular}{|c|c|c|c|c|c|c|c|c|c|c|c|c|}
\hline & \multicolumn{2}{|c|}{ NSTEMI } & \multicolumn{4}{|c|}{$95.0 \% \mathrm{CI}$} & \multicolumn{2}{|c|}{ STEMI } & \multicolumn{4}{|c|}{$95.0 \% \mathrm{CI}$} \\
\hline & $\begin{array}{l}\text { TIMI } \\
\text { flow } 3\end{array}$ & $\begin{array}{c}\text { TIMI } \\
\text { flow } \leq 2\end{array}$ & HR & Lower & Upper & $P$ & $\begin{array}{l}\text { TIMI } \\
\text { flow } 3\end{array}$ & $\begin{array}{c}\text { TIMI } \\
\text { flow } \leq 2\end{array}$ & HR & Lower & Upper & $P$ \\
\hline $\begin{array}{l}\text { Before matching } \\
\text { 5-year }\end{array}$ & $n=1605$ & $n=115$ & & & & & $n=2567$ & $n=2567$ & & & & \\
\hline Primary end-point & $279(17.4)$ & $19(16.5)$ & 0.932 & 0.586 & 1.484 & 0.087 & 407 (15.9) & $66(28.8)^{*}$ & 1.962 & 1.513 & 2.546 & $<0.001$ \\
\hline Cardiac death & $114(7.1)$ & $11(9.6)$ & 1.330 & 0.716 & 2.471 & 0.366 & $187(7.3)$ & $50(21.8)^{*}$ & 3.154 & 2.308 & 4.309 & $<0.001$ \\
\hline Myocardial infarction & $32(2.0)$ & $1(0.9)$ & 0.430 & 0.059 & 3.148 & 0.406 & $64(2.5)$ & $5(2.2)$ & 0.947 & 0.381 & 2.352 & 0.906 \\
\hline Target lesion revascularization & $158(9.8)$ & $8(7.0)$ & 0.708 & 0.348 & 1.439 & 0.340 & $207(8.1)$ & $15(6.6)$ & 0.904 & 0.535 & 1.526 & 0.705 \\
\hline $\begin{array}{l}\text { Propensity-Score matching } \\
\text { 5-year }\end{array}$ & $n=115$ & $n=115$ & & & & & $n=229$ & $n=229$ & & & & \\
\hline Primary end-point & $20(17.4)$ & $19(16.5)$ & 0.931 & 0.496 & 1.744 & 0.822 & $41(17.9)$ & $66(28.8)^{*}$ & 1.692 & 1.145 & 2.498 & 0.008 \\
\hline Cardiac death & $12(10.4)$ & $11(9.6)$ & 0.918 & 0.405 & 2.078 & 0.837 & $26(11.4)$ & $50(21.8)^{*}$ & 1.975 & 1.229 & 3.173 & 0.005 \\
\hline Myocardial infarction & $1(0.9)$ & $1(0.9)$ & 0.775 & 0.048 & 12.412 & 0.857 & $4(1.7)$ & $5(2.2)$ & 1.330 & 0.357 & 4.955 & 0.671 \\
\hline Target lesion revascularization & $8(7.0)$ & $8(7.0)$ & 0.955 & 0.358 & 2.548 & 0.926 & $12(5.2)$ & $15(6.6)$ & 1.372 & 0.642 & 2.933 & 0.414 \\
\hline
\end{tabular}

Data are presented as $\mathrm{n}(\%)$, *Significantly higher rate compared with the postintervention TIMI flow 3

analysis did not demonstrate a significant difference in CD beyond 3 months in STEMI patients $(P=0.267$, Figure 4). However, MI or TLR-free survival rates had no significant differences during the 5-year follow-up ( $P=$ 0.906, $P=0.705$, respectively). On the other hand, the risk for primary end-point in the NSTEMI patients was not significantly different in both groups during the 5-year follow-up $(78.6 \%$ versus $82.1 \% ; P=0.087)$

Predictors of the postprocedural suboptimal coronary flow in MI: Univariable and multivariable logistic regression analyses were performed to identify independent predictors of postinterventional suboptimal coronary flow in STEMI and NSTEMI after PCI. In the multivariable logistic regression model, culprit vessel (LCX, LM disease), and multivessel disease in NSTEMI and LVEF, HDL cholesterol, and $\mathrm{Hb}$ in STEMI were independent predictors of the postprocedural suboptimal coronary flow (Table IV). Compared with the culprit LAD lesion, the prevalence of postprocedural suboptimal flow was not significantly higher in culprit RCA lesion (adjusted HR $=1.063, P=$ 0.602 ) but higher in culprit LM lesion (adjusted HR = 3.182, $P=0.001$ ) in NSTEMI patients. Furthermore, the prevalence of postprocedural suboptimal flow was higher if CAD was extensive in NSTEMI (adjusted HR $=2.031$, $P=0.002$ ). Unexpectedly, the prevalence of postprocedural suboptimal flow was lower in the culprit LCX lesion than the LAD lesion (adjusted HR $=0.544, P=0.001$ ) in NSTEMI patients. Multivariable logistic regression revealed that LVEF and HDL cholesterol are the predictors for the lower prevalence of postprocedural suboptimal flow (adjusted HR $=0.980, P=0.002$; adjusted $\mathrm{HR}=$ $0.984, P=0.025)$ and $\mathrm{Hb}$ is the predictor for the higher prevalence of postprocedural suboptimal flow (adjusted $\mathrm{HR}=1.081, P=0.026)$ in STEMI patients.

\section{Discussion}

This large, multicenter cohort analysis evaluated the outcomes of STEMI and NSTEMI patients during 5 years according to postprocedural TIMI flow. This study included a relatively large number of patients with STEMI and NSTEMI who had undergone primary PCI. The subjects were followed up for 5 years and the results found that suboptimal coronary flow after primary PCI was associated with an increased risk for long-term mortality in STEMI. The mortality risk was high during the first 3 months but became insignificant beyond this time point. Meanwhile, there was no significant difference in the NSTEMI patients with suboptimal coronary flow over the 5 years.

Despite the development of the PCI technique, devices, and medications over the past decades, the incidence of suboptimal flow after primary PCI remains relatively high. This considerable final TIMI flow $\leq 2$ is thought to be due to microvascular dysfunction resulting from vascular constriction, distal microembolization, endothelial dysfunction secondary to endothelial injury, capillary plugging by platelets, neutrophils, and erythrocytes, and intracellular and interstitial edema. ${ }^{12}$ We observed suboptimal coronary flow after primary PCI in $8.2 \%$ and $6.7 \%$ of patients with STEMI and NSTEMI, respectively. This study also identified an association between the final coronary flow and mortality during 5 years after PCI in STEMI and NSTEMI, respectively. The present analysis demonstrated that mortality was significantly lower in the patients with TIMI grade 3 after PCI (19.6\%), as compared with those with TIMI $\leq 2(33.1 \%)$ over 5 years in STEMI patients, whereas mortality did not significantly differ $(21.4 \%$ versus $17.9 \%)$ in NSTEMI patients. As is well known, complete early reperfusion (TIMI grade 3 ) of the infarct-related artery in STEMI is related with mortality benefit. ${ }^{2,413,14)}$ It is consistent that our study showed early survival benefit, but the effect did not persist beyond the early phase of postintervention. This finding underscores the importance of preventing the development of TIMI $\leq 2$ flow as the optimal strategy to improve clinical outcomes and decrease resource utilization in patients carrying out primary PCI since the outcomes are relatively dismal once no-reflow occurs.

Above this, we found that the effect of poor TIMI flow in STEMI is uniquely on death and not the other components of MACE (Table III, Figures 2, 3). 

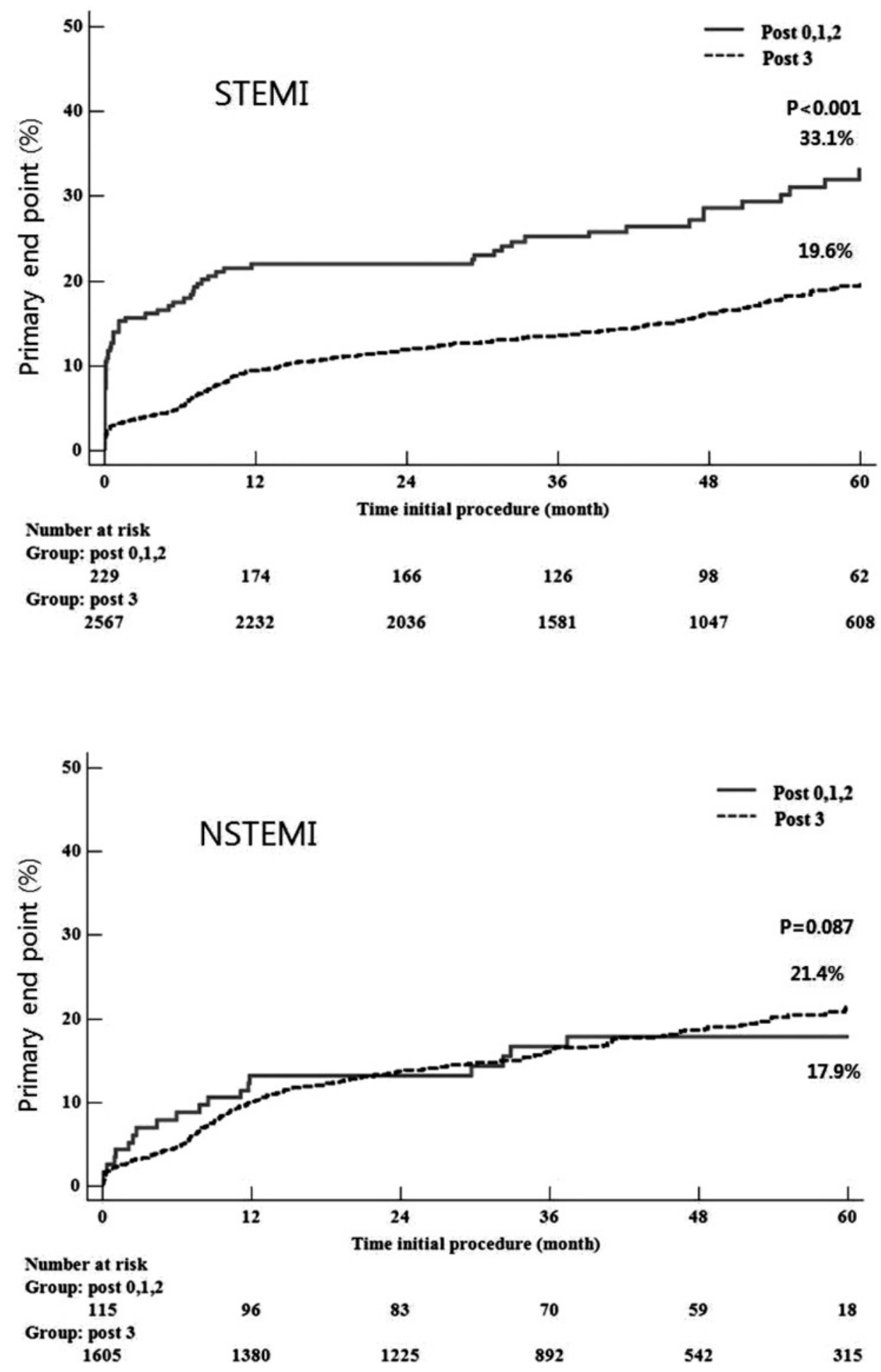

Figure 2. Kaplan-Meier Curve for the primary Endpoint in MI according to post-PCI TIMI flow.

Meanwhile, preTIMI flow of angiography in NSTEMI remains controversial. Although some previous papers contended that preprocedural TIMI flow was an independent predictor for survival in NSTEMI patients, ${ }^{15-17)}$ others reported that preTIMI flow in NSTEMI did not ap- pear to affect survival or mortality.,18,19)

In this regard, our study has an important implication. We focused on post-epicardial flow after PCI, irrespective of preTIMI flow and it indicated that postprocedural coronary flow showed no significant impact on 

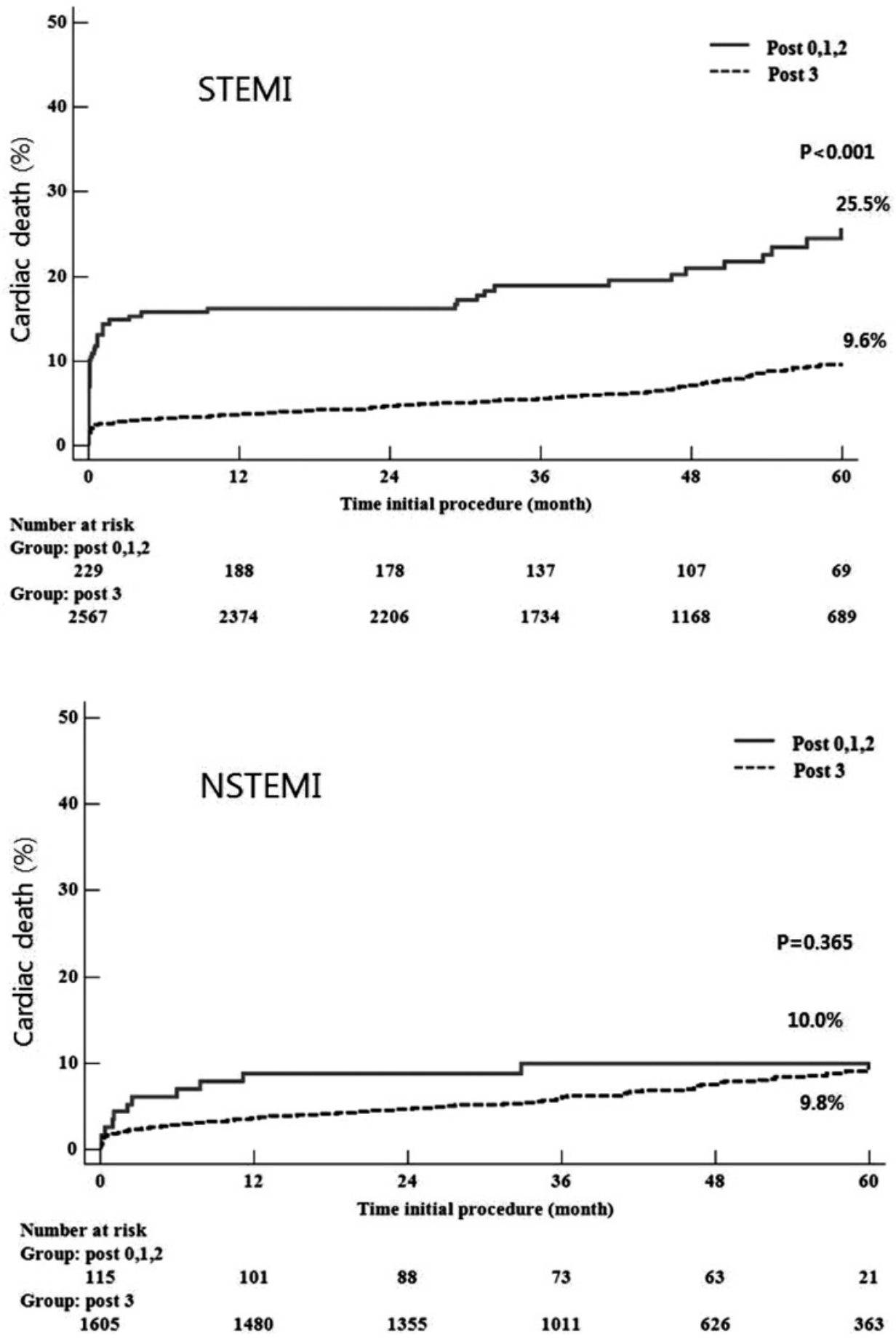

Figure 3. Kaplan-Meier Curves for Cardiac Death in MI according to post-PCI TIMI flow

long-term mortality as well as short term prognosis in NSTEMI patients. This result is supported by recent reports that early intervention made no difference in preventing primary outcome as compared with delayed approach. ${ }^{20-22)}$ Although our study was not differentiated in accordance with risk stratification in NSTEMI, it suggests that not only appropriate intervention time but also the presence of complete final coronary flow through any de- vice procedure or medial approach might affect less than expected in NSTEMI patients.

These differences may be explained by pathophysiologic differences between NSTEMI and STEMI. Differences in the culprit lesion of the two states have recently been demonstrated by optical coherence tomography. The incidences of plaque rupture $(70 \%$ versus $47 \%)$, thin-cap fibroatheroma (78\% versus $49 \%)$, and red thrombus $(78 \%$ 

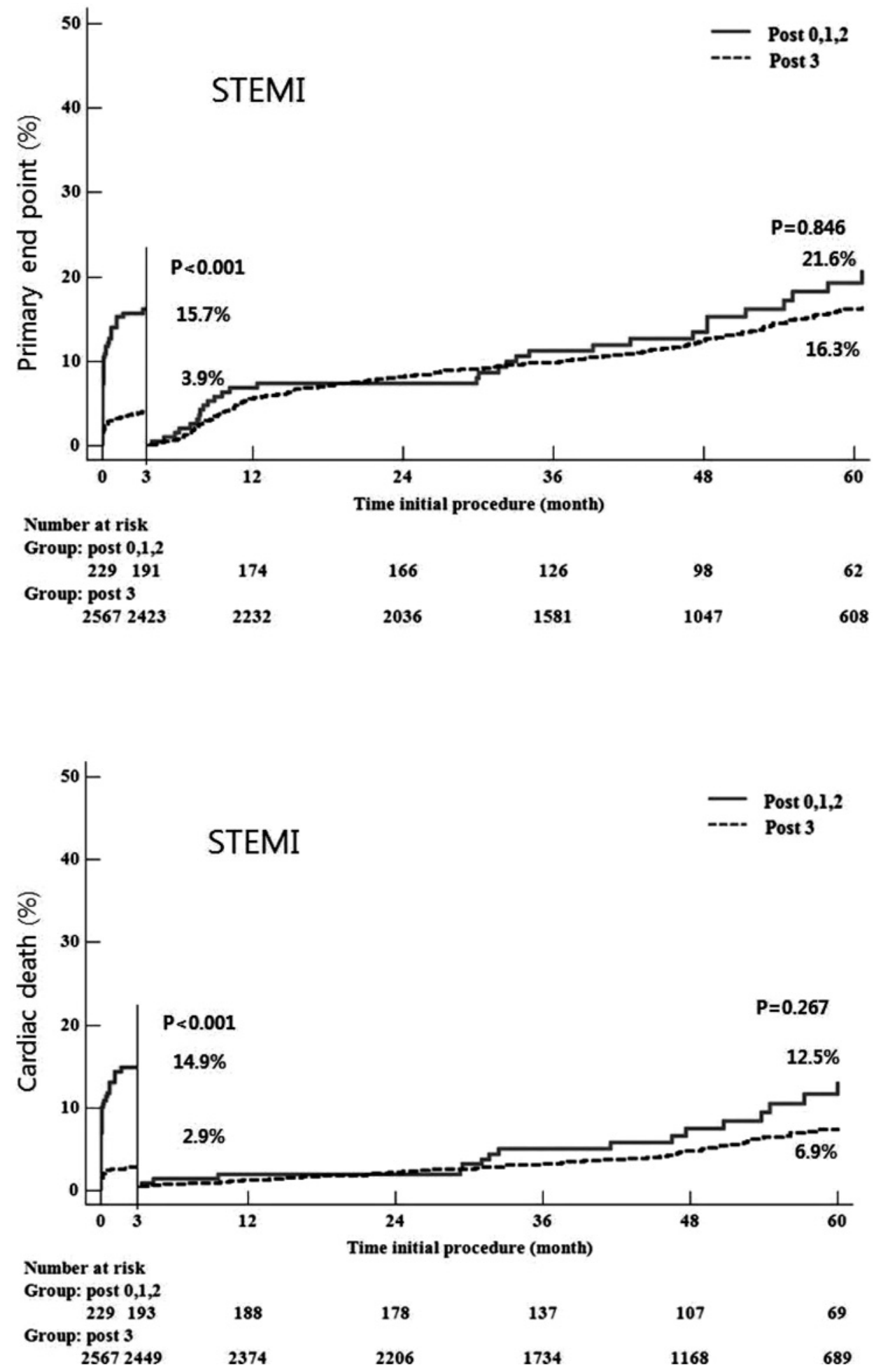

Figure 4. Kaplan-Meier Curve and 3-month landmark analysis for the primary end-point in MI according to post-PCI TIMI flow

versus $49 \%$ ) were significantly higher in STEMI, compared to NSTEMI patients, and they had a larger ruptured cavity area than NSTEMI patients. ${ }^{23)}$ Therefore, as damages by infarct transmurality are not relatively serious in
NSTEMI compared to STEMI, postinterventional coronary flow could have less influences on overall survival benefit. And, there is a potential for early hazard due to intervention on unstable plaques with fresh thrombus. ${ }^{24-26)}$ 
Table IV. Independent Predictors of Postprocedural Suboptimal Flow by Multivariable Logistic Regression Analysis

\begin{tabular}{|c|c|c|c|c|c|c|c|c|c|}
\hline \multirow{2}{*}{ NSTEMI } & \multirow{2}{*}{ OR } & \multicolumn{2}{|c|}{$95 \% \mathrm{CI}$} & \multirow{2}{*}{$P$} & \multirow{2}{*}{ STEMI } & \multirow{2}{*}{ OR } & \multicolumn{2}{|c|}{$95 \% \mathrm{CI}$} & \multirow{2}{*}{$P$} \\
\hline & & Lower & Upper & & & & Lower & Upper & \\
\hline Culprit vessel & & & & 0.002 & Ejection fraction & 0.980 & 0.968 & 0.993 & 0.002 \\
\hline LCX versus LAD & 0.544 & 0.304 & 0.975 & 0.001 & HDL cholesterol & 0.984 & 0.971 & 0.998 & 0.025 \\
\hline RCA versus LAD & 1.063 & 0.678 & 1.666 & 0.602 & Hemoglobin & 1.081 & 1.009 & 1.157 & 0.026 \\
\hline $\mathrm{LM}$ versus $\mathrm{LAD}$ & 3.182 & 1.444 & 7.012 & 0.001 & & & & & \\
\hline $\begin{array}{l}\text { Extent of CAD (multi vessel) } \\
\text { versus single vessel }\end{array}$ & 2.031 & 1.288 & 3.204 & 0.002 & & & & & \\
\hline
\end{tabular}

CI indicates confidence interval; HR, hazard ratio; CAD, coronary artery disease; and HDL, high-density lipoprotein. Significant variables $(P<0.05)$ analysed in multivariable logistic regression model in NSTEMI: age, gender, Killip classification, smoking, culprit vessel, multivessel disease, LVEF, total cholesterol, LDL cholesterol, use of statin Significant variables $(P<$ 0.05 ) analysed in multivariable logistic regression model in STEMI: age, gender, Killip classification, smoking, culprit vessel, LVEF, fasting blood sugar, total cholesterol, triglyceride, HDL cholesterol, hsCRP, hemoglobin, clopidogrel.

Conversely, a relative delayed strategy in NSTEMI might offer benefits through plaque passivation by optical medical treatment followed by intervention on more stable plaques. $^{27,28)}$

Also, the role of ischemic preconditioning could offer substantial protection in NSTEMI patients. Subendocardial viability in NSTEMI patients is thought to be less dependent on collateral circulation and thus more likely to be protected by preconditioning. ${ }^{29)}$ Preconditioning does not prevent or preclude the development of myocardial necrosis, but rather protects the heart by slowing or delaying myocyte death. ${ }^{30,31)}$

In addition, several heterogeneous characteristics of NSTEMI might have an influence on clinical outcome in our study. Angiographically, NSTEMI patients in our study included occluded culprit arteries defined as preTIMI 0 or 1 . Although these patients, often termed "STEMI equivalents", ${ }^{17,32}$ have no ST elevation change in ECG analysis, it has been shown to have worse clinical outcomes compared to NSTEMI patients. ${ }^{16,17)}$ Procedurally, our study underwent emergent, urgent, and delayed PCI except for medial approach in NSTEMI patients. Accordingly, it could affect the clinical outcome in NSTEMI patients. Above these, various medical approaches might be associated with the mortality in NSTEMI patients. Commonly, NSTEMI patients could add anticoagulant therapy (unfractionated heparin, enoxaparin, bivalirudin, or fondaparinus) to antiplatelet therapy for initial management. In our study, although we used various anticoagulant medications in conjunction with standard guideline management, we did not always use anticoagulant therapy for NSTEMI management.

One of the most important findings in this study is the identification of the certain independent predictors for poor angiographic coronary flow after PCI. Previous studies identified DM, age, hypertension, and pre-PCI TIMI flow as the independent predictors for suboptimal coronary flow. ${ }^{33-35)}$

In this study, postprocedural suboptimal coronary flow was diagnosed by coronary angiography and we found that LVEF, HDL cholesterol, Hb in STEMI and culprit vessel type, and the extent of multivessel disease in NSTEMI were the independent predictors for postprocedural suboptimal coronary flow after PCI.
$\mathrm{Hb}$ was independently associated with postintervention TIMI grade $\leq 2$ flow in STEMI patients. It is important to notice that a high $\mathrm{Hb}$ value, a major determinant of whole blood viscosity, predicts cardiovascular events. ${ }^{36}$ ) The relationships between blood viscosity, erythrocyte deformability, coronary risk, and endothelial function modify blood fluidity. A reduced fluidity may limit the microcirculatory flow due to the viscus resistance. ${ }^{37)}$ Coronary anatomy and myocardial blood flow are the major determinants of clinical symptoms and survival in patients with epicardial coronary artery. ${ }^{37,38)}$

LVEF and HDL were found to be relatively benign predictors for postprocedural suboptimal flow despite the presence of other factors in STEMI patients. Although previous studies found a significant relationship between preprocedural TIMI flow and enzymatic infarct size and predischarge $\mathrm{EF},{ }^{18,39)}$ this study showed that postprocedural TIMI flow related with predischarge LVEF. It could be related to more extensive myocardial salvage, and thus better $\mathrm{EF}$ and more limited infarct size. Meanwhile, in case of HDL cholesterol, it could affect atherosclerosis which has been identified as an inflammatory process ${ }^{40)}$ driven by the adaptive ( $\mathrm{T}$ and $\mathrm{B}$ cells) immune system and dendritic cells. ${ }^{41)}$ In our study, a rise of HDL cholesterol might be favorably associated with early inflammatory process for establishment of good coronary flow in STEMI patients. In addition, this study showed that the final coronary flow after PCI was better in LCX territory than in other treated arteries in NSTEMI (HR 0.544 [0.304-0.975], $P=0.001$ ), even after the adjustment for the presence of other factors. In contrast, patients with the left main (LM) disease tended to have the poor final coronary flow (HR 3.182 [1.444-7.012], $P=0.001$ ) more often as seen in LAD lesion (Table IV). Also, even though other culprit vessels did not cause significant differences in mortality risk for 5 years, there were no deaths in postprocedural suboptimal flow group of LCX lesion in NSTEMI patients (Supplemental Table). Large and prospective trials will be necessary to more definitely settle this issue.

There are several potential limitations to our study. First, it was a nonrandomized study, and results are prone to be influenced by selection bias and confounding factors. To control this matter, PS matching was performed 
but the effects of hidden bias affected by the unmeasured confounders were not completely excluded. Second, it is also possible that the rates of suboptimal coronary flow after primary PCI may have been under-reported because TIMI grades were classified by each operator. Myocardial perfusion grade or myocardial blush would have provided further diagnostic information. Third, the limited number of individuals in postprocedural suboptimal coronary flow groups of the patients with STEMI and NSTEMI may have affected the outcomes of this study, although appropriate statistical adjustments were attempted. Finally, this trial has an intrinsic limitation itself due to several heterogenic components in the groups in terms of angiographical, procedural, and medical aspects.

\section{Conclusion}

This long-term trial was conducted to evaluate the differences in long-term prognosis between two MI groups according to post-TIMI flow. The development of suboptimal coronary flow in patients with STEMI after primary PCI was associated with an increased risk for long-term mortality. And we need to take notice that poor postcoronary TIMI flow is uniquely associated with cardiac death, not the other components of MACE. Meanwhile, post-TIMI flow in patients with NSTEMI did not have any long-term effects on survival. Our results suggest that cautious assessment for early risk stratification should be taken into consideration according to initial MI patients' characteristics and may provide guidance in future trials for timing and aggressiveness of revascularization in ACS.

\section{Disclosure}

Conflict of interest: None of the authors have any conflicts of interest to declare.

\section{References}

1. Ross AM, Simoons ML, et al. The effects of tissue plasminogen activator, streptokinase, or both on coronary-artery patency, ventricular function, and survival after acute myocardial infarction. The GUSTO Angiographic Investigators. N Engl J Med 1993; 329: $1615-22$.

2. Vogt A, von Essen R, Tebbe U, Feuerer W, Appel KF, Neuhaus KL. Impact of early perfusion status of the infarct-related artery on short-term mortality after thrombolysis for acute myocardial infarction: retrospective analysis of four German multicenter studies. J Am Coll Cardiol 1993; 21: 1391-5.

3. Zijlstra F, de Boer MJ, Hoorntje JC, Reiffers S, Reiber JH, Suryapranata $\mathrm{H}$. A comparison of immediate coronary angioplasty with intravenous streptokinase in acute myocardial infarction. $\mathrm{N}$ Engl J Med 1993; 328: 680-4.

4. Zijlstra F, Hoorntje JC, de Boer MJ, et al. Long-term benefit of primary angioplasty as compared with thrombolytic therapy for acute myocardial infarction. N Engl J Med 1999; 341: 1413-9.

5. Kenner MD, Zajac EJ, Kondos GT, et al. Ability of the noreflow phenomenon during an acute myocardial infarction to predict left ventricular dysfunction at one-month follow-up. Am J Cardiol 1995; 76: 861-8.

6. Cura FA, L'Allier PL, Kapadia SR, et al. Predictors and prognosis of suboptimal coronary blood flow after primary coronary angioplasty in patients with acute myocardial infarction. Am J Cardiol 2001; 88: 124-8.

7. Ndrepepa G, Mehilli J, Schulz S, et al. Prognostic significance of epicardial blood flow before and after percutaneous coronary intervention in patients with acute coronary syndromes. J Am Coll Cardiol 2008; 52: 512-7.

8. Uyarel H, Ayhan E, Cicek G, et al. Suboptimal coronary blood flow after primary percutaneous coronary intervention for acute myocardial infarction: incidence, a simple risk score, and prognosis. Coron Artery Dis 2012; 23: 98-104.

9. Puymirat E, Taldir G, Aissaoui N, et al. Use of invasive strategy in non-ST-segment elevation myocardial infarction is a major determinant of improved long-term survival: FAST-MI (French Registry of Acute Coronary Syndrome). JACC Cardiovasc Interv 2012; 5: 893-902.

10. Smith SC Jr, Dove JT, Jacobs AK, et al. ACC/AHA guidelines for percutaneous coronary intervention (revision of the 1993 PTCA guidelines)-executive summary: a report of the American College of Cardiology/American Heart Association task force on practice guidelines (Committee to revise the 1993 guidelines for percutaneous transluminal coronary angioplasty) endorsed by the Society for Cardiac Angiography and Interventions. Circulation 2001; 103: 3019-41.

11. Chesebro JH, Knatterud G, Roberts R, et al. Thrombolysis in Myocardial Infarction (TIMI) Trial, Phase I: A comparison between intravenous tissue plasminogen activator and intravenous streptokinase. Clinical findings through hospital discharge. Circulation 1987; 76: 142-54.

12. Rezkalla SH, Kloner RA. No-reflow phenomenon. Circulation 2002; 105: 656-62. (Review)

13. Anderson JL, Karagounis LA, Becker LC, Sorensen SG, Menlove RL. TIMI perfusion grade 3 but not grade 2 results in improved outcome after thrombolysis for myocardial infarction. Ventriculographic, enzymatic, and electrocardiographic evidence from the TEAM-3 Study. Circulation 1993; 87: 1829-39.

14. Anderson JL, Karagounis LA, Califf RM. Metaanalysis of five reported studies on the relation of early coronary patency grades with mortality and outcomes after acute myocardial infarction. Am J Cardiol 1996; 78: 1-8.

15. Kim MC, Ahn Y, Rhew SH, et al. Impact of total occlusion of an infarct-related artery on long-term mortality in acute non-STelevation myocardial infarction patients who underwent early percutaneous coronary intervention. Int Heart J 2012; 53: 160-4.

16. Bahrmann P, Rach J, Desch S, Schuler GC, Thiele H. Incidence and distribution of occluded culprit arteries and impact of coronary collaterals on outcome in patients with non-ST-segment elevation myocardial infarction and early invasive treatment strategy. Clin Res Cardiol 2011; 100: 457-67.

17. Wang TY, Zhang M, Fu Y, et al. Incidence, distribution, and prognostic impact of occluded culprit arteries among patients with non-ST-elevation acute coronary syndromes undergoing diagnostic angiography. Am Heart J 2009; 157: 716-23.

18. De Luca G, Brener SJ, Mehran R, et al. Implications of preprocedural TIMI flow in patients with non ST-segment elevation acute coronary syndromes undergoing percutaneous coronary revascularization: insights from the ACUITY trial. Int $\mathrm{J}$ Cardiol 2013; 167: 727-32.

19. Warren J, Mehran R, Yu J, et al. Incidence and impact of totally occluded culprit coronary arteries in patients presenting with non-ST-segment elevation myocardial infarction. Am J Cardiol 2015; 115: 428-33.

20. Mehta SR, Granger CB, Boden WE, et al. Early versus delayed invasive intervention in acute coronary syndromes. N Engl $\mathrm{J}$ Med 2009; 360: 2165-75.

21. Montalescot G, Cayla G, Collet JP, et al. Immediate vs delayed intervention for acute coronary syndromes: a randomized clinical trial. JAMA 2009; 302: 947-54.

22. Navarese EP, De Servi S, Gibson CM, et al. Early vs. delayed invasive strategy in patients with acute coronary syndromes without ST-segment elevation: a meta-analysis of randomized 
studies. QJM 2011; 104: 193-200. (Review)

23. Ino Y, Kubo T, Tanaka A, et al. Difference of culprit lesion morphologies between ST-segment elevation myocardial infarction and non-ST-segment elevation acute coronary syndrome: an optical coherence tomography study. JACC Cardiovasc Interv 2011; 4: 76-82.

24. de Feyter PJ, van den Brand M, Laarman GJ, van Domburg R, Serruys PW, Suryapranata H. Acute coronary artery occlusion during and after percutaneous transluminal coronary angioplasty. Frequency, prediction, clinical course, management, and follow-up. Circulation 1991; 83: 927-36.

25. Boden WE, O'Rourke RA, Crawford MH, et al. Outcomes in patients with acute non-Q-wave myocardial infarction randomly assigned to an invasive as compared with a conservative management strategy. Veterans Affairs Non-Q-Wave Infarction Strategies in Hospital (VANQWISH) Trial Investigators. N Engl J Med 1998; 338: 1785-92.

26. Schühlen H, Kastrati A, Dirschinger J, et al. Intracoronary stenting and risk for major adverse cardiac events during the first month. Circulation 1998; 98: 104-11.

27. van den, Brand M, Laarman GJ, Steg PG, et al. Assessment of coronary angiograms prior to and after treatment with abciximab, and the outcome of angioplasty in refractory unstable angina patients. Angiographic results from the CAPTURE trial. Eur Heart J 1999; 20: 1572-8.

28. Zhao XQ, Theroux P, Snapinn SM, Sax FL. Intracoronary thrombus and platelet glycoprotein IIb/IIIa receptor blockade with tirofiban in unstable angina or non-Q-wave myocardial infarction. Angiographic results from the PRISM-PLUS trial (Platelet receptor inhibition for ischemic syndrome management in patients limited by unstable signs and symptoms). PRISMPLUS Investigators. Circulation 1999; 100: 1609-15.

29. Papadopoulos CE, Karvounis HI, Gourasas IT, Parharidis GE, Louridas GE. Evidence of ischemic preconditioning in patients experiencing first non-ST-segment elevation myocardial infarction (NSTEMI). Int J Cardiol 2003; 92: 209-17.

30. Przyklenk K, Kloner RA. Preconditioning: a balanced perspective. Br Heart J 1995; 74: 575-7.

31. Przyklenk K, Kloner RA. Ischemic preconditioning: exploring the paradox. Prog Cardiovasc Dis 1998; 40: 517-47. (Review)

32. Stribling WK, Kontos MC, Abbate A, et al. Left circumflex occlusion in acute myocardial infarction (from the National Cardiovascular Data Registry). Am J Cardiol 2011; 108: 959-63.

33. Wu J, Pride YB, Frederick PD, Gibson CM. Association of in- itial thrombolysis in myocardial infarction flow grade with mortality among patients with ST-segment elevation myocardial infarction undergoing primary percutaneous coronary intervention: a National Registry of Myocardial Infarction-5 (NRMI-5) analysis. Am Heart J 2011; 162: 178-83.

34. Shiraishi J, Kohno Y, Sawada T, et al. Predictors of nonoptimal coronary flow after primary percutaneous coronary intervention with stent implantation for acute myocardial infarction. J Cardiol 2010; 55: 217-23.

35. Mehta RH, Harjai KJ, Cox D, et al. Clinical and angiographic correlates and outcomes of suboptimal coronary flow inpatients with acute myocardial infarction undergoing primary percutaneous coronary intervention. J Am Coll Cardiol 2003; 42: 173946.

36. Levenson J, Gariepy J, Del-Pino M, Salomon J, Denarie N, Simon A. Association of plasma viscosity and carotid thickening in a French working cohort. Am J Hypertens 2000; 13: 753-8.

37. Leschke M, Motz W, Strauer BE. Hemorheologic therapy applications in coronary heart disease. Wien Med Wochenschr (German) 1986; 136: 17-24.

38. Majmudar MD, Murthy VL, Shah RV, et al. Quantification of coronary flow reserve in patients with ischaemic and nonischaemic cardiomyopathy and its association with clinical outcomes.. Eur Heart J Cardiovasc Imaging 2015; 16: 900-9.

39. Brodie BR, Stuckey TD, Hansen C, Muncy D. Benefit of coronary reperfusion before intervention on outcomes after primary angioplasty for acute myocardial infarction. Am J Cardiol 2000; 85: 13-8.

40. Ross R. Atherosclerosis-an inflammatory disease. N Engl J Med 1999; 340: 115-26. (Review)

41. Ait-Oufella H, Sage AP, Mallat Z, Tedgui A. Adaptive (T and B cells) immunity and control by dendritic cells in atherosclerosis. Circ Res 2014; 114: 1640-60. (Review)

\section{Supplemental Files}

Supplemental Table

Please see supplemental file;

https://www.jstage.jst.co.jp/article/ihj/58/5/58_16-448/_article/supple ment 